\title{
Parameterized Linear Temporal Logics Meet Costs: Still not Costlier than LTL $^{\star}$
}

\author{
Martin Zimmermann \\ Reactive Systems Group, Saarland University, 66123 Saarbrücken, Germany \\ \{zimmermann\}@react.uni-saarland.de
}

\begin{abstract}
We continue the investigation of parameterized extensions of Linear Temporal Logic (LTL) that retain the attractive algorithmic properties of LTL: a polynomial space model checking algorithm and a doubly-exponential time algorithm for solving games. Alur et al. and Kupferman et al. showed that this is the case for Parametric LTL (PLTL) and PROMPT-LTL respectively, which have temporal operators equipped with variables that bound their scope in time. Later, this was also shown to be true for Parametric LDL (PLDL), which extends PLTL to be able to express all $\omega$-regular properties.

Here, we generalize PLTL to systems with costs, i.e., we do not bound the scope of operators in time, but bound the scope in terms of the cost accumulated during time. Again, we show that model checking and solving games for specifications in PLTL with costs is not harder than the corresponding problems for LTL. Finally, we discuss PLDL with costs and extensions to multiple cost functions.
\end{abstract}

\section{Introduction}

Parameterized linear temporal logics address a serious shortcoming of Lineartemporal Logic (LTL): LTL is not able to express timing constraints, e.g., while $\mathbf{G}(q \rightarrow \mathbf{F} p)$ expresses that every request $q$ is eventually answered by a response $p$, the waiting time between requests and responses might diverge. This is typically not the desired behavior, but cannot be ruled out by LTL.

To overcome this shortcoming, Alur et al. introduced parameterized LTL [1], which extends LTL with parameterized operators of the form $\mathbf{F}_{\leq x}$ and $\mathbf{G}_{\leq y}$, where $x$ and $y$ are variables. The formula $\mathbf{G}\left(q \rightarrow \mathbf{F}_{\leq x} p\right)$ expresses that every request is answered within an arbitrary, but fixed number of steps $\alpha(x)$. Here, $\alpha$ is a variable valuation, a mapping of variables to natural numbers. Typically, one is interested in whether a PLTL formula is satisfied with respect to some variable valuation. For example, the model checking problem asks whether a given transition system satisfies a given PLTL specification $\varphi$ with respect to some $\alpha$, i.e., whether every path satisfies $\varphi$ with respect to $\alpha$. Similarly, solving

\footnotetext{
* Supported by the project "TriCS" (ZI 1516/1-1) of the German Research Foundation (DFG).
} 
infinite games amounts to determining whether there is an $\alpha$ such that Player 0 has a strategy such that every play that is consistent with the strategy satisfies the winning condition with respect to $\alpha$. Alur et al. showed that the PLTL model checking problem is PSPACE-complete. Kupferman et al. later considered PROMPT-LTL 18, which can be seen as the fragment of PLTL without the parameterized always operator, and showed that PROMPT-LTL model checking is still PSPACE-complete and that PROMPT-LTL realizability, an abstract notion of infinite game, is 2ExPTIME-complete. While the results of Alur et al. relied on involved pumping arguments, the results of Kupferman et al. where all based on the so-called alternating-color technique, which basically allows to reduce PROMPT-LTL to LTL. Furthermore, the result on realizability was extended to infinite games on graphs [28, again using the alternating-color technique.

Another serious shortcoming of LTL (and its parameterized variants) is their expressiveness: LTL is equi-expressive to first-order logic with order and thus not as expressive as $\omega$-regular expressions. This shortcoming was addressed by a long line of temporal logics [14|19|25|26|27] with regular expressions, finite automata, or grammar operators to obtain the full expressivity of the $\omega$-regular languages. One of these logics is Linear Dynamic Logic (LDL), which has temporal operators $\langle r\rangle$ and $[r]$, where $r$ is a regular expression. For example, the formula $\left[r_{0}\right]\left(q \rightarrow\left\langle r_{1}\right\rangle p\right)$ holds in a word $w$, if every request at a position $n$ such that $w_{0} \cdots w_{n}$ matches $r_{0}$, there is a position $n^{\prime} \geq n$ such that $p$ holds at $n^{\prime}$ and $w_{n} \cdots w_{n^{\prime}}$ matches $r_{1}$. Intuitively, the diamond operator corresponds to the eventuality of LTL, but is guarded by a regular expression. Dually, the box-operator is a guarded always. Although LDL is more expressive than LTL, its algorithmic properties are similar: model checking is PSPACE-complete and solving games is 2ExPTIME-complete [25].

All these logics tackle one shortcoming, but not both simultaneously. This was achieved for the first time by adding parameterized operators to LDL. The logic, called parameterized LDL (PLDL), has additional operators $\langle r\rangle_{\leq x}$ and $[r]_{\leq y}$ with the expected semantics: the variables bound the scope of the operator. And even this logic, which has parameters and is more expressive than LTL, model checking is still PSPACE-complete and solving games is 2ExPTIME-complete. Again, these problems were solved by an application of the alternating-color technique. One has to overcome some technicalities, but the general proof technique is the same as for PROMPT-LTL.

The decision problems for the parameterized logics mentioned above are boundedness problems, e.g., one asks for an upper bound on the waiting times between requests and responses in case of the formula $\mathbf{G}\left(q \rightarrow \mathbf{F}_{\leq x} p\right)$. Recently, more general boundedness problems in logics and automata received a lot of attention to obtain decidable quantitative extensions of monadic second-order logic and better synthesis algorithms. in general, boundedness problems are undecidable for automata with counters, but become decidable if the acceptance conditions can refer to boundedness properties of the counters, but the transition relation has no access to counter values. Recent advances include logics and automata with bounds [36], satisfiability algorithms for these logics [457/24], 
and regular cost-functions [13. However, these formalisms, while very expressive, are intractable and thus not suitable for verification and synthesis. Thus, less expressive formalisms were studied that appear more suitable for practical applications, e.g., finitary parity [11, parity with costs [17, energy-parity [10], meanpayoff-parity [12, consumption games [8], and the use of weighted automata for specifying quantitative properties [2]. In particular, the parity condition with cost is defined in graphs whose edges are weighted by natural numbers (interpreted as costs) and requires the existence of a bound $b$ such that almost every occurrence of an odd color is followed by an occurrence of a larger even color such that the cost between these positions is at most $b$. Although strictly stronger than the classical parity condition, solving parity games with costs is as hard as solving parity games [17.

Our contribution: We investigate parameterized temporal logics in a weighted setting similar to the one of parity conditions with costs: our graphs are equipped with cost-functions that label the edges with natural numbers and parameterized operators are now evaluated with respect to cost instead of time, i.e., the parameters bound the accumulated cost instead of the elapsed time. Thus, the formula $\mathbf{G}\left(q \rightarrow \mathbf{F}_{\leq x} p\right)$ requires that every request $q$ is answered with cost at most $\alpha(x)$. We show the following results about PLTL with costs (cPLTL):

First, we refined the alternating-color technique to the cost-setting, which requires to tackle some technical problems induced by the fact that accumulated cost, unlike time, does not increase in every step, e.g., if an edge with cost zero is traversed.

Second, we show that Kupferman et al.'s proofs based on the alternatingcolor technique can be adapted to the cost-setting as well. For model-checking, we again obtain PSPACE-completeness while solving games is still 2ExPTIMEcomplete.

Third, we consider PLDL with costs (cPLDL), which is defined as expected. Again, the complexity does not increase: model checking is PSPACE-complete while solving games is 2ExPTIME-complete.

Fourth, we generalize both logics to a setting with multiple cost-functions. Now, the parameterized temporal operators have another parameter $i$ that determines the cost-function under which they are evaluated. Even these extensions do not increase complexity: model checking is again PSPACE-complete while solving games is still 2ExPTIME-complete.

Fifth, we also investigate model checking and solving games as an optimization problem, which is a very natural view on the problems, i.e., we are interested in computing the optimal variable valuation such that a given system satisfies a given specification. For cPLTL and cPLDL, we show that the model checking optimization problem can be solved in polynomial space while the optimization problem for infinite games can be solved in triply-exponential time. These results are similar to the ones obtained for PLTL 28. In particular, the exponential gap between the decision and the optimization variant of solving infinite games exists already for PLTL.

All proofs omitted due to space restrictions can be found in the appendix. 


\section{Parametric LTL with Costs}

Let $\mathcal{V}$ be an infinite set of variables and let $P$ be a set of atomic propositions. The formulae of cPLTL are given by the grammar

$$
\varphi::=p|\neg p| \varphi \wedge \varphi|\varphi \vee \varphi| \mathbf{X} \varphi|\varphi \mathbf{U} \varphi| \varphi \mathbf{R} \varphi\left|\mathbf{F}_{\leq z} \varphi\right| \mathbf{G}_{\leq z} \varphi
$$

where $p \in P$ and $z \in \mathcal{V}$. We use the derived operators tt $:=p \vee \neg p$ and $\mathrm{ff}:=p \wedge \neg p$ for some fixed $p \in P, \mathbf{F} \varphi:=\operatorname{tt} \mathbf{U} \varphi$, and $\mathbf{G} \varphi:=\operatorname{ff} \mathbf{R} \varphi$. Furthermore, we use $p \rightarrow \psi$ and $\neg p \rightarrow \psi$ as shorthand for $\neg p \vee \varphi$ and $p \vee \varphi$, respectively. Additional derived operators are introduced on page 5 .

The set of subformulae of a cPLTL formula $\varphi$ is denoted by $\operatorname{cl}(\varphi)$ and we define the size of $\varphi$ to be the cardinality of $\operatorname{cl}(\varphi)$. Furthermore, we define $\operatorname{var}_{\mathbf{F}}(\varphi)=\left\{z \in \mathcal{V} \mid \mathbf{F}_{\leq z} \psi \in \operatorname{cl}(\varphi)\right\}$ to be the set of variables parameterizing eventually operators in $\varphi, \operatorname{var}_{\mathbf{G}}(\varphi)=\left\{z \in \mathcal{V} \mid \mathbf{G}_{\leq z} \psi \in \operatorname{cl}(\varphi)\right\}$ to be the set of variables parameterizing always operators in $\varphi$, and set $\operatorname{var}(\varphi)=\operatorname{var}_{\mathbf{F}}(\varphi) \cup \operatorname{var}_{\mathbf{G}}(\varphi)$. From now on, we denote variables $\operatorname{in} \operatorname{var}_{\mathbf{F}}(\varphi)$ by $x$ and $\operatorname{variables~}_{\operatorname{in}} \operatorname{var}_{\mathbf{G}}(\varphi)$ by $y$, if the formula $\varphi$ is clear from context.

cPLTL is evaluated on so-called cost-traces (traces for short) of the form

$$
w=w_{0} c_{0} w_{1} c_{1} w_{2} c_{2} \cdots \in\left(2^{P} \cdot \mathbb{N}\right)^{\omega},
$$

which encode the evolution of the system in terms of the atomic propositions that hold true in each time instance, and the cost of changing the system state. The cost of the trace $w$ is defined as $\operatorname{cst}(w)=\sum_{j \geq 0} c_{j}$, which might be infinite. A finite cost-trace is required to begin and end with an element of $2^{P}$. The $\operatorname{cost} \operatorname{cst}(w)$ of a finite cost-trace $w=w_{0} c_{0} w_{1} c_{1} \cdots c_{n-1} w_{n}$ is defined as $\operatorname{cst}(w)=$ $\sum_{j=0}^{n-1} c_{j}$.

Furthermore, we require the existence of a special atomic proposition $\kappa$ such that all cost-traces satisfy $c_{j}>0$ if and only if $\kappa \in w_{j+1}$, i.e., $\kappa$ indicates that the last step had non-zero cost. We use the proposition $\kappa$ to reason about costs: for example, we are able to express whether a trace has cost zero or $\infty$. In the following, we will ensure that all our systems only allow traces that satisfy this assumption.

Also, to evaluate formulas we need to instantiate the variables parameterizing the temporal operators. To this end, we define a variable valuation to be a mapping $\alpha: \mathcal{V} \rightarrow \mathbb{N}$. Now, we can define the model relation between a costtrace $w=w_{0} c_{0} w_{1} c_{1} w_{2} c_{2} \cdots$, a position $n$ of $w$, a variable valuation $\alpha$, and a cPLTL formula as follows:

$-(w, n, \alpha) \models p$ if and only if $p \in w_{n}$,

$-(w, n, \alpha) \models \neg p$ if and only if $p \notin w_{n}$,

- $(w, n, \alpha) \models \varphi \wedge \psi$ if and only if $(w, n, \alpha) \models \varphi$ and $(w, n, \alpha) \models \psi$,

$-(w, n, \alpha) \models \varphi \vee \psi$ if and only if $(w, n, \alpha) \models \varphi$ or $(w, n, \alpha) \models \psi$,

$-(w, n, \alpha) \models \mathbf{X} \varphi$ if and only if $(w, n+1, \alpha) \models \varphi$,

$-(w, n, \alpha) \models \varphi \mathbf{U} \psi$ if and only if there exists a $j \geq 0$ such that $(w, n+j, \alpha) \models \psi$ and $(w, n+k, \alpha) \models \varphi$ for every $k$ in the range $0 \leq k<j$, 
- $(w, n, \alpha) \models \varphi \mathbf{R} \psi$ if and only if for every $j \geq 0$ : either $(w, n+j, \alpha) \models \psi$ or there exists a $k$ in the range $0 \leq k<j$ such that $(w, n+k, \alpha) \models \varphi$,

- $(w, n, \alpha) \models \mathbf{F}_{\leq z} \varphi$ if and only if there exists a $j \geq 0$ with $\operatorname{cst}\left(w_{n} c_{n} \cdots c_{n+j-1} w_{n+j}\right) \leq \alpha(z)$ such that $(w, n+j, \alpha) \models \varphi$, and

- $(w, n, \alpha) \models \mathbf{G}_{\leq z} \varphi$ if and only if for every $j \geq 0$ with $\operatorname{cst}\left(w_{n} c_{n} \cdots c_{n+j-1} w_{n+j}\right) \leq \alpha(z):(w, n+j, \alpha) \models \varphi$.

Note that we recover the semantics of PLTL as the special case where every $c_{n}$ is equal to one.

For the sake of brevity, we write $(w, \alpha) \models \varphi$ instead of $(w, 0, \alpha) \models \varphi$ and say that $w$ is a model of $\varphi$ with respect to $\alpha$. For variable-free formulas, we even drop the $\alpha$ and write $w \models \alpha$.

As usual for parameterized temporal logics, the use of variables has to be restricted: bounding eventually and always operators by the same variable leads to an undecidable satisfiability problem [1].

Definition 1. A cPLTL formula $\varphi$ is well-formed, if $\operatorname{var}_{\mathbf{F}}(\varphi) \cap \operatorname{var}_{\mathbf{G}}(\varphi)=\emptyset$.

In the following, we only consider well-formed formulas and omit the qualifier "well-formed". We consider the following fragments of cPLTL. Let $\varphi$ be a cPLTL formula:

$-\varphi$ is an LTL formula, if $\operatorname{var}(\varphi)=\emptyset$.

$-\varphi$ is a cPLTL $_{\mathbf{F}}$ formula, if $\operatorname{var}_{\mathbf{G}}(\varphi)=\emptyset$.

$-\varphi$ is a $\mathrm{cPLTL}_{\mathbf{G}}$ formula, if $\operatorname{var}_{\mathbf{F}}(\varphi)=\emptyset$.

Example 1.

1. The formula $\mathbf{G}\left(q \rightarrow \mathbf{F}_{\leq x} p\right)$ is satisfied with respect to $\alpha$, if every request (a position where $q$ holds) is followed by a response (a position where $p$ holds) such that the cost of the infix between the request and the response is at most $\alpha(x)$.

2. The (max-) parity condition with costs 17 can be expressed in cPLTL via

$$
\mathbf{F G}\left(\bigwedge_{c \in\{1,3, \ldots, d-1\}}\left(c \rightarrow \mathbf{F}_{\leq x} \bigvee_{c^{\prime} \in\{c+1, c+3, \ldots, d\}} c^{\prime}\right)\right)
$$

where $d$ is the maximal color, which we assume w.l.o.g. to be even. However, the Streett condition with costs [17] cannot be expressed in cPLTL, as it is defined with respect to multiple cost functions, one for each Streett pair. We extend cPLTL to multiple cost functions in Section 7.

As for PLTL, one can also parameterize the until and the release operator and also consider bounds of the form " $>z$ ". However, this does not increase expressiveness of the logic. Formally, we define

- $(w, n, \alpha) \models \varphi \mathbf{U}_{\leq z} \psi$ if and only if there exists a $j \geq 0$ with $\operatorname{cst}\left(w_{n} c_{n} \cdots c_{n+j-1} w_{n+j}\right) \leq \alpha(z)$ such that $(w, n+j, \alpha) \models \psi$ and $(w, n+$ $k, \alpha) \models \varphi$ for every $k$ in the range $0 \leq k<j$, 
- $(w, n, \alpha) \models \varphi \mathbf{R}_{\leq z} \psi$ if and only if for every $j \geq 0$ with $\operatorname{cst}\left(w_{n} c_{n} \cdots c_{n+j-1} w_{n+j}\right) \leq \alpha(z):(w, n+j, \alpha) \models \psi$ or there exists a $k$ in the range $0 \leq k<j$ such that $(w, n+k, \alpha) \models \varphi$,

- $(w, n, \alpha) \models \mathbf{F}_{>z} \varphi$ if and only if there exists a $j \geq 0$ with $\operatorname{cst}\left(w_{n} c_{n} \cdots c_{n+j-1} w_{n+j}\right)>\alpha(z)$ such that $(w, n+j, \alpha) \models \varphi$, and

- $(w, n, \alpha) \models \mathbf{G}_{>z} \varphi$ if and only if for every $j \geq 0$ with $\operatorname{cst}\left(w_{n} c_{n} \cdots c_{n+j-1} w_{n+j}\right)>\alpha(z)$ satisfies $(w, n+j, \alpha) \models \varphi$.

- $(w, n, \alpha) \models \varphi \mathbf{U}_{>z} \psi$ if and only if there exists a $j \geq 0$ with $\operatorname{cst}\left(w_{n} c_{n} \cdots c_{n+j-1} w_{n+j}\right)>\alpha(z)$ such that $(w, n+j, \alpha) \models \psi$ and $(w, n+$ $k, \alpha) \models \varphi$ for every $k$ in the range $0 \leq k<j$, and

- $(w, n, \alpha) \models \varphi \mathbf{R}_{>z} \psi$ if and only if for every $j \geq 0$ with $\operatorname{cst}\left(w_{n} c_{n} \cdots c_{n+j-1} w_{n+j}\right)>\alpha(z):(w, n+j, \alpha) \models \psi$ or there exists a $k$ in the range $0 \leq k<j$ such that $(w, n+k, \alpha) \models \varphi$.

Let $\varphi \equiv \psi$ denote equivalence of the formulas $\varphi$ and $\psi$, i.e., for every $w$, every $n$, and every $\alpha$, we have $(w, n, \alpha) \models \varphi$ if and only if $(w, n, \alpha) \models \psi$. Then, we have the following equivalences (which also restrict the use of variables as defined in Definition (1):

$$
\begin{array}{ll}
-\varphi \mathbf{U}_{\leq z} \psi \equiv \varphi \mathbf{U} \psi \wedge \mathbf{F}_{\leq z} \psi & -\mathbf{G}_{>z} \varphi \equiv \mathbf{F}_{\leq z} \mathbf{G X}(\neg \kappa \vee \mathbf{G} \varphi) \\
-\varphi \mathbf{R}_{\leq z} \psi \equiv \varphi \mathbf{R} \psi \vee \mathbf{G}_{\leq z} \psi & -\varphi \mathbf{U}_{>z} \psi \equiv \mathbf{G}_{\leq z}(\varphi \wedge \mathbf{F X}(\kappa \wedge \varphi \mathbf{U} \psi)) \\
-\mathbf{F}_{>z} \varphi \equiv \mathbf{G}_{\leq z} \mathbf{F X}(\kappa \wedge \mathbf{F} \varphi) & -\varphi \mathbf{R}_{>z} \psi \equiv \mathbf{F}_{\leq z}(\varphi \vee \mathbf{G X}(\neg \kappa \vee \varphi \mathbf{R} \psi))
\end{array}
$$

Note that we defined cPLTL formulae to be in negation normal form. Nevertheless, a negation can be pushed to the atomic propositions using the duality of the operators. Thus, we can define the negation of a cPLTL formula.

Lemma 1. For every cPLTL formula $\varphi$ there exists an efficiently constructible cPLTL formula $\neg \varphi$ s.t.

1. $(w, n, \alpha) \models \varphi$ if and only if $(w, n, \alpha) \not \models \neg \varphi$ for every $w$, every $n$, and every $\alpha$,

2. $|\neg \varphi|=|\varphi|$.

3. If $\varphi$ is well-formed, then so is $\neg \varphi$.

4. If $\varphi$ is an LTL formula, then so is $\neg \varphi$.

5. If $\varphi$ is a cPLTL $L_{\mathbf{F}}$ formula, then $\neg \varphi$ is a cPLTL $L_{\mathbf{G}}$ formula and vice versa.

Proof. We construct $\neg \varphi$ by induction over the construction of $\varphi$ using the dualities of the operators:

$$
\begin{aligned}
& -\neg(p)=\neg p \\
& -\neg(\varphi \wedge \psi)=(\neg \varphi) \vee(\neg \psi) \\
& -\neg(\varphi \mathbf{U} \psi)=\neg \varphi \mathbf{R} \neg \psi \\
& -\neg\left(\mathbf{F}_{\leq x} \varphi\right)=\mathbf{G}_{\leq x} \neg \varphi
\end{aligned}
$$

$$
\begin{aligned}
& -\neg(\neg p)=p \\
& -\neg(\varphi \vee \psi)=(\neg \varphi) \wedge(\neg \psi) \\
& -\neg(\varphi \mathbf{R} \psi)=\neg \varphi \mathbf{U} \neg \psi \\
& -\neg\left(\mathbf{G}_{\leq y} \varphi\right)=\mathbf{F}_{\leq y} \neg \varphi
\end{aligned}
$$

The latter four claims of Lemma 1 follow from the definition of $\neg \varphi$ while the first one can be shown by a straightforward induction over the construction of $\varphi$. 
Another important property of parameterized logics is monotonicity: increasing (decreasing) the values of parameterized eventuality operators (parameterized always operators) preserves satisfaction.

Lemma 2. Let $\varphi$ be a cPLTL formula and let $\alpha$ and $\beta$ be variable valuations satisfying $\beta(x) \geq \alpha(x)$ for every $x \in \operatorname{var}_{\mathbf{F}}(\varphi)$ and $\beta(y) \leq \alpha(y)$ for every $y \in$ $\operatorname{var}_{\mathbf{G}}(\varphi)$. If $(w, \alpha) \models \varphi$, then $(w, \beta) \models \varphi$.

Especially, if we are interested in checking whether a formula is satisfied with respect to some $\alpha$, we can always recursively replace every subformula $\mathbf{G}_{\leq y} \psi$ by $\psi \vee \mathbf{X}(\neg \kappa \mathbf{U}(\neg \kappa \wedge \psi))$, as this is equivalent to $\mathbf{G}_{\leq y} \psi$ with respect to every variable valuation mapping $y$ to zerd 1 , which is the smallest possible value for $y$. Note that we have to ignore the current truth value of $\kappa$, as it indicates the cost of the last transition, not the cost of the next one.

\section{The Alternating-Color Technique for Costs}

Fix a fresh atomic proposition $p \notin P$. We say that a cost-trace

$$
w^{\prime}=w_{0}^{\prime} c_{0}^{\prime} w_{1}^{\prime} c_{1}^{\prime} w_{2}^{\prime} c_{2}^{\prime} \cdots \in\left(2^{P \cup\{p\}} \cdot \mathbb{N}\right)^{\omega}
$$

is a coloring of a cost trace $w=w_{0} c_{0} w_{1} c_{1} w_{2} c_{2} \cdots \in\left(2^{P} \cdot \mathbb{N}\right)^{\omega}$, if $w_{n}^{\prime} \cap P=w_{n}$ and $c_{n}^{\prime}=c_{n}$ for every $n$, i.e., $w^{\prime}$ and $w$ only differ in the truth values of the new proposition $p$. A position $n$ is a changepoint of $w^{\prime}$, if $n=0$ or if the truth value of $p$ in $w_{n-1}^{\prime}$ and $w_{n}^{\prime}$ differs. A block of $w^{\prime}$ is an infix $w_{n}^{\prime} c_{n}^{\prime} \cdots w_{n+j}^{\prime}$ of $w^{\prime}$ such that $n$ and $n+j+1$ are successive changepoints. If a coloring has only finitely many changepoints, then we refer to its suffix starting at the last changepoint as its tail, i.e., the coloring is the concatenation of a finite number of blocks and its tail.

Let $k \in \mathbb{N}$. We say that $w^{\prime}$ is $k$-bounded if every block and its tail (if it has one) has cost at most $k$. Dually, we say that $w^{\prime}$ is $k$-spaced, if every block has cost at least $k$. Note that we do not have a requirement on the cost of the tail in this case.

Given a $\mathrm{cPLTL}_{\mathbf{F}}$ formula $\varphi$, let $\operatorname{rel}(\varphi)$ denote the LTL formula obtained from $\varphi$ by recursively replacing every subformula $\mathbf{F}_{\leq x} \psi$ by

$$
(p \rightarrow p \mathbf{U}(\neg p \mathbf{U r e l}(\psi))) \wedge(\neg p \rightarrow \neg p \mathbf{U}(p \mathbf{U r e l}(\psi))) .
$$

Intuitively, the relativized formula requires $\operatorname{rel}(\psi)$ to be satisfied within at most one changepoint. On bounded and spaced colorings, $\varphi$ and $\operatorname{rel}(\varphi)$ are "equivalent".

Lemma 3 (cp. Lemma 2.1 of [18]). Let $w$ be a cost-trace and let $\varphi$ be a $c P L T L_{\mathbf{F}}$ formula.

\footnotetext{
${ }^{1}$ Here, we use our assumption on $\kappa$ indicating the sign of the cost.
} 
1. Let $(w, \alpha) \models \varphi$ for some variable valuation $\alpha$. Then, $w^{\prime} \models \operatorname{rel}(\varphi)$ for every $(k+1)$-spaced coloring $w^{\prime}$ of $w$, where $k=\max _{x \in \operatorname{var}(\varphi)} \alpha(x)$.

2. Let $w^{\prime} \models \operatorname{rel}(\varphi)$ for some $k$-bounded coloring $w^{\prime}$ of $w$. Then, $(w, \alpha) \models \varphi$, where $\alpha(x)=2 k$ for every $x$.

Proof. Note that $w$ and its colorings coincide on their cost. Hence, when speaking about the cost of an infix or suffix, we do not have to specify whether we refer to $w$ or to a coloring of $w$.

1.) Fix a $(k+1)$-spaced coloring $w^{\prime}$ of $w$, where $k=\max _{x \in \operatorname{var}(\varphi)} \alpha(x)$. We show that $(w, n, \alpha) \models \varphi$ implies $\left(w^{\prime}, n\right) \models \operatorname{rel}(\varphi)$ by induction over the construction of $\varphi$.

The only non-trivial case is the one of a parameterized eventuality: thus, assume $(w, n, \alpha) \models \mathbf{F}_{\leq x} \psi$, i.e., there is a $j$ with $\operatorname{cst}\left(w_{n} c_{n} \cdots c_{n+j-1} w_{n+j}\right) \leq \alpha(x)$ and $(w, n+j, \alpha) \models \bar{\psi}$. By induction hypothesis, we have $\left(w^{\prime}, n+j\right) \models \operatorname{rel}(\psi)$. As $w^{\prime}$ is $(k+1)$-spaced, i.e., the cost of each block is at least $k+1$, there is at most one changepoint between (and including) the positions $n$ and $n+j-1$ in $w^{\prime}$. Hence, $\left.\left(w^{\prime}, n\right) \models p \mathbf{U}(\neg p \mathbf{U r e l}(\psi))\right)$, if $p \in w_{n}^{\prime}$, and $\left.\left(w^{\prime}, n\right) \models \neg p \mathbf{U}(p \mathbf{U r e l}(\psi))\right)$ otherwise. Thus, $\left(w^{\prime}, n\right) \models \operatorname{rel}\left(\mathbf{F}_{\leq x} \psi\right)$.

2.) Dually, fix a $k$-bounded coloring $w^{\prime}$ of $w$ and define the variable valuation $\alpha$ with $\alpha(x)=2 k$ for every $x$. We show that $\left(w^{\prime}, n\right) \models \operatorname{rel}(\varphi)$ implies $(w, n, \alpha) \models \varphi$ by induction over the construction of $\varphi$.

Again, the only non-trivial case is the one of a parameterized eventuality: thus, let $\left(w^{\prime}, n\right) \models \operatorname{rel}\left(\mathbf{F}_{<x} \psi\right)$. We assume $\left(w^{\prime}, n\right) \models p$ (the other case is dual). Then, we have $\left(w^{\prime}, n\right) \models p \mathbf{U}(\neg p \mathbf{U} \operatorname{rel}(\psi))$, i.e., $\operatorname{rel}(\psi)$ is satisfied at some position $n+j$ such that there is at most one changepoint between (and including) the positions $n$ and $n+j-1$ in $w^{\prime}$. As $w^{\prime}$ is $k$-bounded, this implies that the cost of the infix $w_{n} c_{n} \cdots w_{n+j}$ is bounded by $2 k$. Furthermore, applying the induction hypothesis yields $(w, n+j, \alpha) \models \psi$. Hence, $(w, n, \alpha) \models \mathbf{F}_{\leq x} \psi$.

\section{Model Checking}

A transition system $\mathcal{S}=\left(S, s_{I}, E, \ell, \mathrm{cst}\right)$ consists of a finite directed graph $(S, E)$, an initial state $s_{I} \in S$, a labeling function $\ell: S \rightarrow 2^{P}$, and a cost function cst: $E \rightarrow \mathbb{N}$. We assume that every state has at least one successor to spare us from dealing with finite paths. Recall our requirement on cost-traces having a distinguished atomic property $\kappa$ indicating the sign of the cost of the previous transition. Thus, we require $\mathcal{S}$ to satisfy the following property: if $\kappa \in \ell\left(v^{\prime}\right)$, then $\operatorname{cst}\left(v, v^{\prime}\right)>0$ for every edge $\left(v, v^{\prime}\right) \in E$ leading to $v^{\prime}$. Dually, if $\kappa \notin \ell\left(v^{\prime}\right)$, then $\operatorname{cst}\left(v, v^{\prime}\right)=0$ for every edge $\left(v, v^{\prime}\right) \in E$

A path through $\mathcal{S}$ is a sequence $\pi=s_{0} s_{1} s_{2} \cdots$ satisfying $s_{0}=s_{I}$ and $\left(s_{n}, s_{n+1}\right) \in E$ for every $n$. Its cost-trace $\operatorname{tr}(\pi)$ is defined as

$$
\operatorname{tr}(\pi)=\ell\left(s_{0}\right) \operatorname{cst}\left(s_{0}, s_{1}\right) \ell\left(s_{1}\right) \operatorname{cst}\left(s_{1}, s_{2}\right) \ell\left(s_{2}\right) \operatorname{cst}\left(s_{2}, s_{3}\right) \cdots,
$$

which satisfies our assumption on the proposition $\kappa$. 
The transition system $\mathcal{S}$ satisfies a cPLTL formula $\varphi$ with respect to a variable valuation $\alpha$, if the trace of every path through $\mathcal{S}$ satisfies $\varphi$ with respect to $\alpha$. The cPLTL model checking problem asks, given a transition system $\mathcal{S}$ and a cPLTL formula $\varphi$, whether $\mathcal{S}$ satisfies $\varphi$ with respect to some $\alpha$.

Theorem 1. The cPLTL model checking problem is PSPACE-complete.

The proof we give below is a generalization of the one for PROMPT-LTL by Kupferman et al. [18. We begin by showing PSPACE-membership. First note that we can restrict ourselves to CPLTL $_{\mathbf{F}}$ formulas: given a cPLTL formula $\varphi$, let $\varphi^{\prime}$ denote the formula obtained by recursively replacing every subformula $\mathbf{G}_{\leq y} \psi$ by $\psi \vee \mathbf{X}(\neg \kappa \mathbf{U}(\neg \kappa \wedge \psi))$. Due to Lemma 2 and the discussion below it, every transition system $\mathcal{S}$ satisfies $\varphi$ with respect to some $\alpha$ if and only if $\mathcal{S}$ satisfies $\varphi^{\prime}$ with respect to some $\alpha^{\prime}$.

Next, we show how to apply the alternating-color: recall that the classical algorithm for LTL model checking searches for a fair path, i.e., one that visits infinitely many accepting states, in the product of $\mathcal{S}$ with a Büchi automaton recognizing the models of the negated specification. If such a path exists, then $\mathcal{S}$ does not satisfy the specification, as the fair path contains a path $\pi$ through $\mathcal{S}$ and an accepting run of the automaton on its trace, i.e., the trace does not satisfy the specification. If there is no such fair path, then $\mathcal{S}$ satisfies the specification.

For cPLTL we have to find such a path for every $\alpha$ in order to show that $\mathcal{S}$ does not satisfy the specification with respect to any $\alpha$. To this end, one relativizes the cPLTL $_{\mathbf{F}}$ specification as described in Section 3 and builds an automaton for the negation of the relativized formula in conjunction with a formula that ensures that every ultimately periodic model is both $k$-bounded and $k^{\prime}$-spaced for some appropriate $k$ and $k^{\prime}$. Then, we search for a pumpable fair path in the product of the system and the Büchi automaton recognizing the models of the negated specification, which is non-deterministically labeled by $p$. Applying Lemma 3 and pumping a fair path through the product appropriately yields a counterexample for every $\alpha$. Thus, model checking is reduced to finding a pumpable fair path. Let us stress again that this algorithm is similar to the one for PROMPT-LTL, we just have to pay attention to some intricacies stemming from the fact that we want to bound the cost, not the waiting time: there might be paths with finite cost, which have to be dealt with appropriately.

Recall that $p$ is the distinguished atomic proposition used to relativize cPLTL formulas. A colored Büchi graph with costs $\left(V, v_{I}, E, \ell\right.$, cst, $\left.F\right)$ consists of a finite direct graph $(V, E)$, an initial vertex $v_{I}$, a labeling function $\ell: V \rightarrow 2^{\{p\}}$, a costfunction cst: $E \rightarrow \mathbb{N}$, and a set $F \subseteq V$ of accepting vertices. A path $v_{0} v_{1} v_{2} \ldots$ is pumpable, if each of its blocks induced by $p$ contains a vertex repetition such that the cycle formed by the repetition has non-zero cost 2 . Note that we do not have a requirement on the cost of the tail, if the path has one. The path is fair, if it visits $F$ infinitely often. The pumpable non-emptiness problem asks, given a colored Büchi graph with costs, whether it has an initial pumpable fair path.

\footnotetext{
${ }^{2}$ Note that our definition is more involved than the one of Kupferman et al., since we require a cycle with non-zero cost instead of any circle.
} 
Lemma 4. If a colored Büchi graph with costs has an initial pumpable fair path, then also one of the form $\pi_{0} \pi_{1}^{\omega}$ with $\left|\pi_{0} \pi_{1}\right| \in \mathcal{O}\left(n^{2}\right)$, where $n$ is the number of vertices of the graph.

Proof. Let $\pi$ be an arbitrary initial pumpable fair path. First, assume it has only finitely many changepoints. If there are two blocks that start with the same vertex, then we can remove all blocks in between and obtain another initial pumpable fair path. Thus, we can assume that $\pi$ has at most $n$ blocks. Furthermore, the length of each block can be bounded by $\mathcal{O}(n)$ by removing cycles while retaining the state repetition with non-zero cost and at least one accepting vertex (provided the block has one). Now, consider the tail: by removing infixes one can find a cycle of length at most $n$ containing an accepting vertex and a path of length at most $n$ leading from the last changepoint to a vertex on the cycle. Hence, we define $\pi_{0}$ to be the prefix containing all blocks and the path leading to the cycle and define $\pi_{1}$ to be the cycle. Then, we have $\left|\pi_{0} \pi_{1}\right| \in \mathcal{O}\left(n^{2}\right)$ and $\pi_{0} \pi_{1}^{\omega}$ is an initial pumpable fair path.

On the other hand, if $\pi$ contains infinitely many changepoints, then we can remove blocks and shorten other blocks as described above until we have constructed a prefix $\pi_{0} \pi_{1}$ such that $\pi_{0} \pi_{1}^{\omega}$ has the desired properties. In this case, we can assume that the first position of $\pi_{1}$ is a changepoint by "rotating" $\pi_{1}$ appropriately and appending a suitable prefix of it to $\pi_{0}$.

Let $\mathcal{S}=\left(S, s_{I}, E, \ell\right.$, cst $)$ be a transition system and let $\varphi$ be a $\mathrm{cPLTL}_{\mathbf{F}}$ formula. Furthermore, consider the LTL formula $\chi=(\mathbf{G F} p \wedge \mathbf{G F} \neg p) \leftrightarrow \mathbf{G F} \kappa$, which is satisfied by a cost-trace, if the trace has infinitely many changepoints if and only if 3 it has cost $\infty$. Now, let $\mathfrak{A}=\left(Q, 2^{P \cup\{p\}}, q_{I}, \delta, F\right)$ be a nondeterministic Büchi automaton recognizing the models of the LTL formula $\neg \operatorname{rel}(\varphi) \wedge \chi$, which we can pick such that its number of states is bounded exponentially in $|\varphi|$. Now, define the colored Büchi graph with costs $\mathcal{S} \times \mathfrak{A}=(S \times Q \times$ $\left.2^{\{p\}},\left(s_{I}, q_{I}, \emptyset\right), E^{\prime}, \ell^{\prime}, \operatorname{cst}^{\prime}, F^{\prime}\right)$ where

- $\left((s, q, C),\left(s^{\prime}, q^{\prime}, C^{\prime}\right)\right) \in E^{\prime}$ if and only if $\left(s, s^{\prime}\right) \in E$ and $q^{\prime} \in \delta(q, \ell(s) \cup C)$,

$-\ell(s, q, C)=C$,

$-\operatorname{cst}^{\prime}\left((s, q, C),\left(s^{\prime}, q^{\prime}, C^{\prime}\right)\right)=\operatorname{cst}\left(s, s^{\prime}\right)$, and

$-F^{\prime}=S \times F \times 2^{\{p\}}$.

Lemma 5. [cp. Lemma 4.2 of [18]] $\mathcal{S}$ does not satisfy $\varphi$ with respect to any $\alpha$ if and only if $\mathcal{S} \times \mathfrak{A}$ has an initial pumpable fair path.

Proof. Let $\mathcal{S}$ not satisfy $\varphi$ with respect to any variable valuation. Fix $k=$ $(|S| \cdot|Q|+3) \cdot W$, where $W$ is the largest cost in $\mathcal{S}$, and define the valuation $\alpha$ by $\alpha(x)=2 k$ for every $x$. As $\mathcal{S}$ does not satisfy $\varphi$ with respect to $\alpha$, there is a path $\pi$ through $\mathcal{S}$ with $(\operatorname{tr}(\pi), \alpha) \not \forall \varphi$. Thus, due to Lemma 312, every $k$-bounded coloring of $w$ does not satisfy $\operatorname{rel}(\varphi)$.

Now, let $w^{\prime}$ be a $k$-bounded and $(k-W)$-spaced coloring of $\operatorname{tr}(\pi)$ which starts with $p$ not holding true. Such a coloring can always be constructed, as $W$ is the

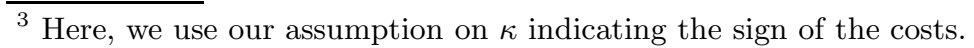


largest cost appearing in $\mathcal{S}$. Note that $w^{\prime}$ satisfies $\chi$ by construction. Thus, we have $w^{\prime} \models \neg \operatorname{rel}(\varphi) \wedge \chi$, i.e., there is an accepting run $q_{0} q_{1} q_{2} \cdots$ of $\mathfrak{A}$ on $w^{\prime}$. Consider the path

$$
\left(s_{0}, q_{0}, w_{0}^{\prime} \cap\{p\}\right)\left(s_{1}, q_{1}, w_{1}^{\prime} \cap\{p\}\right)\left(s_{2}, q_{2}, w_{2}^{\prime} \cap\{p\}\right) \cdots
$$

where $s_{0} s_{1} s_{2} \cdots=\pi$, which is fair by construction. We claim that it is pumpable: consider a block, which is $(k-W)$-spaced. Thus, it contains at least $|S| \cdot|Q|+2$ many edges with non-zero cost, enough to enforce a vertex repetition with nonzero cost in between. To this end, one takes the sets $V_{j}$ of vertices visited between the $j$-th and the $(j+1)$-th edge with non-zero cost (including the $j$-th edge). This yields $|S| \cdot|Q|+1$ non-empty sets of vertices of $\mathcal{S} \times \mathfrak{A}$ that coincide in their third component, as we are within one block. However, there are only $|S| \cdot|Q|$ many such vertices, which yields the desired repetition.

Now, consider the second implication and let $\alpha^{\prime}$ be an arbitrary variable valuation. We show that $\mathcal{S}$ does not satisfy $\varphi$ with respect to $\alpha^{\prime}$. Due to Lemma2, it is sufficient to show that $\mathcal{S}$ does not satisfy $\varphi$ with respect to the valuation $\alpha$ mapping every variable to $k=\min _{x \in \operatorname{var}(\varphi)} \alpha^{\prime}(x)$.

Fix an initial pumpable fair path of $\mathcal{S} \times \mathfrak{A}$. It has a vertex repetition in every block such that the induced cycle has non-zero cost. We pump each such cycle $k+1$ times to obtain the path

$$
\left(s_{0}, q_{0}, C_{0}\right)\left(s_{1}, q_{1}, C_{1}\right)\left(s_{2}, q_{2}, C_{2}\right) \cdots .
$$

By construction, $\pi=s_{0} s_{1} s_{2} \cdots$ is a path through $\mathcal{S}$ and

$$
w^{\prime}=\left(\ell\left(s_{0}\right) \cup C_{0}\right)\left(\ell\left(s_{1}\right) \cup C_{1}\right)\left(\ell\left(s_{2}\right) \cup C_{2}\right) \cdots
$$

is a coloring of its trace $\operatorname{tr}(\pi)$. Also, $q_{0} q_{1} q_{2} \cdots$ is an accepting run of $\mathfrak{A}$ on $w^{\prime}$, i.e., $w^{\prime} \models \neg \operatorname{rel}(\varphi) \wedge \chi$. Lastly, $w^{\prime}$ is $(k+1)$-spaced by construction, as the cost-function of $\mathcal{S} \times \mathfrak{A}$ is induced by the one of $\mathcal{S}$.

Assume towards a contradiction that $\mathcal{S}$ satisfies $\varphi$ with respect to $\alpha$, which implies $(\operatorname{tr}(\pi), \alpha) \models \varphi$. Applying Lemma 311 yields that every $(k+1)$-spaced coloring of $\operatorname{tr}(\pi)$ satisfies $\operatorname{rel}(\varphi)$. However, $w^{\prime}$ is a $(k+1)$-spaced coloring which satisfies $\neg \operatorname{rel}(\varphi)$, i.e., we have derived the desired contradiction.

Now, we are ready to prove Theorem 1 .

Proof. PSPACE-hardness holds already for LTL [23, which is a fragment of cPLTL. Membership is witnessed by the following algorithm: check whether the colored Büchi graph $\mathcal{S} \times \mathfrak{A}$ has an initial pumpable fair path, which is correct due to Lemma 5 . But as the graph is of exponential size, it has to be constructed and tested for non-emptiness on-the-fly.

Due to Lemma 4 it suffices to check for the existence of an ultimately periodic path $\pi_{0} \pi_{1}^{\omega}$ such that $\left|\pi_{0} \pi_{1}\right| \leq n \in \mathcal{O}(|\mathcal{S} \times \mathfrak{A}|)$, i.e., $n$ is exponential in the size of $\varphi$ and linear in the size of $\mathcal{S}$. To this end, one guesses a vertex $v$ (the first vertex of $\pi_{1}$ ) and checks the following reachability properties: 
1. Is $v$ reachable from $v_{I}$ via a path where each block contains a cycle with non-zero cost?

2. Is $v$ reachable from $v$ via a non-empty path that visits an accepting vertex and which either has no changepoint or where each block contains a cycle with non-zero cost? In this case, we also require that $v$ and the last vertex on the path from $v_{I}$ to $v$ guessed in item 1,) differ on their third component in order to make $v$ a changepoint. This spares us from having a block that spans $\pi_{0}$ and $\pi_{1}$.

All these reachability problems can be solved in non-deterministic polynomial space, as a successor of a vertex of $\mathcal{S} \times \mathfrak{A}$ can be guessed and verified in polymonial time and the length of the paths to be guessed is bounded by $n$, which can be represented with polynomially many bits.

Furthermore, by applying both directions of the proof of Lemma 5, we obtain an exponential upper bound on the values of a satisfying variable valuation, if one exists. This is asymptotically tight, as one can already show exponential lower bounds for PROMPT-LTL [18.

Corollary 1. Fix a transition system $\mathcal{S}$ and a cPLTL-formula $\varphi$ such that $\mathcal{S}$ satisfies $\varphi$ with respect to some $\alpha$. Then, $\mathcal{S}$ satisfies $\varphi$ with respect to a valuation that is bounded exponentially in the size of $\varphi$ and linearly in the number of states of $\mathcal{S}$ and the maximal cost in $\mathcal{S}$.

Dually, using pumping arguments one can show the existence of an exponential variable valuation that witnesses whether a given CPLTL $_{\mathbf{G}}$ specification is satisfied with respect to every variable valuation.

Lemma 6. Fix a transition system $\mathcal{S}$ and a $c P L T L_{\mathbf{G}}$-formula $\varphi$ such that $\mathcal{S}$ does not satisfy $\varphi$ with respect to every $\alpha$. Then, $\mathcal{S}$ does not satisfy $\varphi$ with respect to a valuation that is bounded exponentially in the size of $\varphi$ and linearly in the number of states of $\mathcal{S}$ and the maximal cost in $\mathcal{S}$.

The proof of the preceding Lemma is similar to the one of Lemma 7 in [16].

\section{$5 \quad$ Infinite Games}

An arena $\mathcal{A}=\left(V, V_{0}, V_{1}, v_{I}, E, \ell\right.$, cst $)$ consists of a finite directed graph $(V, E)$, a partition $\left(V_{0}, V_{1}\right)$ of $V$, an initial vertex $v_{I} \in V$, a labeling $\ell: V \rightarrow 2^{P}$, and a cost function cst: $E \rightarrow \mathbb{N}$. Again, we assume that every vertex has at least one successor to avoid dealing with finite paths. Also, we again ensure our requirement on the proposition $\kappa$ to indicate the sign of the costs in a cost-trace: if $\kappa \in \ell\left(v^{\prime}\right)$, then we require $\operatorname{cst}\left(v, v^{\prime}\right)>0$ for every edge $\left(v, v^{\prime}\right) \in E$ leading to $v^{\prime}$. Dually, if $\kappa \notin \ell\left(v^{\prime}\right)$, then $\operatorname{cst}\left(v, v^{\prime}\right)=0$ for every edge $\left(v, v^{\prime}\right) \in E$.

A play $\rho=\rho_{0} \rho_{1} \rho_{2} \cdots$ is a path through $\mathcal{A}$ starting in $v_{I}$ and its costtrace $\operatorname{tr}(\rho)$ is defined as

$$
\operatorname{tr}(\rho)=\ell\left(\rho_{0}\right) \operatorname{cst}\left(\rho_{0}, \rho_{1}\right) \ell\left(\rho_{1}\right) \operatorname{cst}\left(\rho_{1}, \rho_{2}\right) \ell\left(\rho_{2}\right) \operatorname{cst}\left(\rho_{2}, \rho_{3}\right) \cdots .
$$


A strategy for Player $i \in\{0,1\}$ is a mapping $\sigma: V^{*} V_{i} \rightarrow V$ satisfying $(v, \sigma(w v)) \in E$ for every $w \in V^{*}$ and $v \in V_{i}$. A play $\rho$ is consistent with $\sigma$ if $\rho_{n+1}=\sigma\left(\rho_{0} \cdots \rho_{n}\right)$ for every $n$ with $\rho_{n} \in V_{i}$.

A cPLDL game $\mathcal{G}=(\mathcal{A}, \varphi)$ consists of an arena $\mathcal{A}$ and a winning condition $\varphi$, which is a cPLDL formula. A strategy $\sigma$ for Player 0 is winning with respect to some variable valuation $\alpha$, if the trace of every play that is consistent with $\sigma$ satisfies the winning condition $\varphi$ with respect to $\alpha$.

We are interested in determining whether Player 0 has a winning strategy for a given cPLDL game, and in determining a winning strategy for her if this is the case.

Theorem 2. Determining whether Player 0 has a winning strategy in a given cPLTL game is 2EXPTIME-complete. Furthermore, a winning strategy (if one exists) can be computed in doubly-exponential time.

Our proof technique is a generalization of the one for infinite games with PLTL winning conditions [28, which in turn extended Kupferman et al.'s solution to the PROMPT-LTL realizability problem [18. First, we note that it is again sufficient to consider $\mathrm{CPLTL}_{\mathbf{F}}$ formulas, as we are interested in the existence of a variable valuation (see the discussion below Lemma 2). Next, we apply the alternating-color technique: to this end, we modify the arena to allow Player 0 to produce colorings of plays of the original arena and use the relativized winning condition, i.e., we reduce the problem to a game with LTL winning condition. The winner (and a winning strategy) of such a game can be computed in doubly-exponential time [20121].

To allow for the coloring, we double the vertices of the arena, additionally label one copy with $p$ and the other not, and split every move into two: first, the player whose turn it is picks an outgoing edge, then Player 0 decides in which copy she wants to visit the target, thereby picking the truth value of $p$.

Formally, given an arena $\mathcal{A}=\left(V, V_{0}, V_{1}, v_{I}, E, \ell, \mathrm{cst}\right)$, the extended arena $\mathcal{A}^{\prime}=\left(V^{\prime}, V_{0}^{\prime}, V_{1}^{\prime}, v_{I}^{\prime}, E^{\prime}, \ell^{\prime}, c s t^{\prime}\right)$ consists of

- $V^{\prime}=V \times\{0,1\} \cup E$,

- $V_{0}^{\prime}=V_{0} \times\{0,1\} \cup E$ and $V_{1}^{\prime}=V_{1} \times\{0,1\}$,

$-v_{I}^{\prime}=\left(v_{I}, 0\right)$,

- $E^{\prime}=\left\{((v, 0), e),((v, 1), e),\left(e,\left(v^{\prime}, 0\right)\right),\left(e,\left(v^{\prime}, 1\right)\right) \mid e=\left(v, v^{\prime}\right) \in E\right\}$,

$-\ell^{\prime}(e)=\emptyset$ for every $e \in E$ and $\ell^{\prime}(v, b)=\left\{\begin{array}{ll}\ell(v) & \text { if } b=0, \\ \ell(v) \cup\{p\} & \text { if } b=1,\end{array}\right.$ and

$-\operatorname{cst}^{\prime}\left((v, b),\left(v, v^{\prime}\right)\right)=\operatorname{cst}\left(v, v^{\prime}\right)$ and $\operatorname{cst}^{\prime}\left(\left(v, v^{\prime}\right),\left(v^{\prime}, b^{\prime}\right)\right)=0$.

A path through $\mathcal{A}^{\prime}$ has the form $\left(\rho_{0}, b_{0}\right) e_{0}\left(\rho_{1}, b_{1}\right) e_{1}\left(\rho_{2}, b_{2}\right) \cdots$ for some path $\rho_{0} \rho_{1} \rho_{2} \cdots$ through $\mathcal{A}$, where $e_{n}=\left(\rho_{n}, \rho_{n+1}\right)$ and $b_{n} \in\{0,1\}$. Also, we have $\left|\mathcal{A}^{\prime}\right| \in \mathcal{O}\left(|\mathcal{A}|^{2}\right)$. Note that we use the costs in $\mathcal{A}^{\prime}$ only to argue the correctness of our construction, not to define the winning condition for the game in $\mathcal{A}^{\prime}$.

Note that the additional choice vertices of the form $e \in E$ have to be ignored when it comes to evaluating the winning condition on the trace of a play. 
Thus, we consider games with LTL winning conditions under so-called blinking semantics: Player 0 wins a play $\rho=\rho_{0} \rho_{1} \rho_{2} \cdots$ under blinking semantics, if $\ell\left(\rho_{0}\right) \ell\left(\rho_{2}\right) \ell\left(\rho_{4}\right) \cdots$ satisfies the winning condition $\varphi$; otherwise, Player 1 wins. Winning strategies under blinking semantics are defined as expected. Determining whether Player 0 has a winning strategy for a given game with LTL winning condition under blinking semantics is 2ExPTIME-complete, which can be shown by a slight variation of the proof for LTL games under classical semantics [20 21]. Furthermore, if Player 0 has a winning strategy for such a game, then also a finite-state one of at most doubly-exponential size in $|\varphi|$.

Such a strategy is given by a memory structure $\mathcal{M}=\left(M, m_{I}\right.$, upd) with a finite set $M$ of memory states, an initial memory state $m_{I} \in M$, and an update function upd: $M \times V \rightarrow M$, and by a next-move function nxt: $V_{0} \times M \rightarrow V$ satisfying $(v, \operatorname{nxt}(v, m)) \in E$ for every $m$ and every $v$. The function $\operatorname{upd}^{*}: V^{+} \rightarrow$ $M$ is defined via $\operatorname{upd}^{*}(v)=m_{I}$ and $\operatorname{upd}^{*}(w v)=\operatorname{upd}\left(\operatorname{upd}^{*}(w), v\right)$. Then, the strategy $\sigma$ implemented by $\mathcal{M}$ and nxt is defined by $\sigma(w v)=\operatorname{nxt}\left(v, \operatorname{upd}^{*}(w v)\right)$. The size of $\sigma$ is (slightly abusively) defined as $|M|$.

Given a game $(\mathcal{A}, \varphi)$ with cPLTL $_{\mathbf{F}}$ winning condition $\varphi$, define $\mathcal{A}^{\prime}$ as above and let $\varphi^{\prime}=\operatorname{rel}(\varphi) \wedge \chi$, where $\chi=(\mathbf{G F} p \wedge \mathbf{G F} \neg p) \leftrightarrow \mathbf{G F} \kappa$. Recall that $\chi$ is satisfied by a cost-trace, if the trace has infinitely many changepoints if and only if it has cost $\infty$.

Lemma 7. [cp. Lemma 3.1 of [18]] Player 0 has a winning strategy for $(\mathcal{A}, \varphi)$ with respect to some $\alpha$ if and only if she has a winning strategy for $\left(\mathcal{A}^{\prime}, \varphi^{\prime}\right)$ under blinking semantics.

Proof. Let $\sigma$ be a winning strategy for Player 0 in $(\mathcal{A}, \varphi)$ with respect to some fixed $\alpha$ and define $k=\max _{x \in \operatorname{var}(\varphi)} \alpha(x)$. We define a strategy $\sigma^{\prime}$ for $\mathcal{A}^{\prime}$ as follows:

$$
\sigma^{\prime}\left(\left(\rho_{0}, b_{0}\right)\left(\rho_{0}, \rho_{1}\right) \cdots\left(\rho_{n-1}, \rho_{n}\right)\left(\rho_{n}, b_{n}\right)\right)=\left(\rho_{n}, \sigma\left(\rho_{0} \cdots \rho_{n}\right)\right)
$$

if $\left(\rho_{n}, b_{n}\right) \in V_{0}^{\prime}$, which implies $\rho_{n} \in V_{0}$. Thus, at a non-choice vertex, Player 0 mimics the behavior of $\sigma$. At choice vertices, she alternates between the two copies of the arena every time the cost has exceeded $k+1$ : let

$$
w=\left(\rho_{0}, b_{0}\right)\left(\rho_{0}, \rho_{1}\right) \cdots\left(\rho_{n}, b_{n}\right)\left(\rho_{n}, \rho_{n+1}\right)
$$

be a play prefix ending in a choice vertex and let $n^{\prime} \leq n$ be the last changepoint in $\ell^{\prime}\left(\rho_{0}, b_{0}\right) \cdots \ell^{\prime}\left(\rho_{n}, b_{n}\right)$. Now, we define

$$
\sigma^{\prime}(w)= \begin{cases}\left(\rho_{n+1}, 0\right) & \text { if }\left(\operatorname{cst}\left(\rho_{n^{\prime}} \cdots \rho_{n}\right)<k+1 \text { and } b_{n}=0\right) \text { or } \\ & \left(\operatorname{cst}\left(\rho_{n^{\prime}} \cdots \rho_{n}\right) \geq k+1 \text { and } b_{n}=1\right), \\ \left(\rho_{n+1}, 1\right) & \text { if }\left(\operatorname{cst}\left(\rho_{n^{\prime}} \cdots \rho_{n}\right)<k+1 \text { and } b_{n}=1\right) \text { or } \\ & \left(\operatorname{cst}\left(\rho_{n^{\prime}} \cdots \rho_{n}\right) \geq k+1 \text { and } b_{n}=0\right) .\end{cases}
$$

Let $\rho=\rho_{0} \rho_{1} \rho_{2} \cdots$ be a play in $\mathcal{A}^{\prime}$ that is consistent with $\sigma^{\prime}$ and let

$$
\rho^{\prime}=\rho_{0} \rho_{2} \rho_{4} \cdots=\left(v_{0}, b_{0}\right)\left(v_{1}, b_{1}\right)\left(v_{2}, b_{2}\right) \cdots \text {. }
$$


By definition of $\sigma^{\prime}$, the sequence $v_{0} v_{1} v_{2} \cdots$ is a play in $\mathcal{A}$ that is consistent with $\sigma$ and thus winning for Player 0 with respect to $\alpha$, i.e., $\left(\operatorname{tr}\left(v_{0} v_{1} v_{2} \cdots\right), \alpha\right) \models \varphi$. Also, $w^{\prime}=\ell^{\prime}\left(v_{0}, b_{0}\right) \ell^{\prime}\left(v_{1}, b_{1}\right) \ell^{\prime}\left(v_{2}, b_{2}\right) \cdots$ is a $(k+1)$-spaced coloring of the trace $\operatorname{tr}\left(v_{0} v_{1} v_{2} \cdots\right)$. Hence, $w^{\prime} \models \varphi^{\prime}$ due to Lemma 311. Finally, $w^{\prime}$ satisfies $\chi$ by construction. Thus, $\sigma^{\prime}$ is a winning strategy for $\left(\mathcal{A}^{\prime}, \varphi^{\prime}\right)$ under blinking semantics.

Now, let $\sigma^{\prime}$ be a winning strategy for Player 0 in $\left(\mathcal{A}^{\prime}, \varphi^{\prime}\right)$ which we can assume (w.l.o.g.) to be implemented by $\mathcal{M}^{\prime}=\left(M^{\prime}, m_{I}^{\prime}\right.$, upd $\left.{ }^{\prime}\right)$ and some nextmove function nxt' such that $\left|M^{\prime}\right|$ is doubly-exponential in $|\varphi|$. We define a strategy $\sigma$ for $\mathcal{A}$ by simulating a play in $\mathcal{A}^{\prime}$ that is consistent with $\sigma^{\prime}$.

To this end, define the memory structure $\mathcal{M}=\left(M, m_{I}\right.$, upd) for $\mathcal{A}$ with $M=(V \times\{0,1\}) \times M^{\prime}, m_{I}=\left((v, 0), m_{I}^{\prime}\right)$, and

$$
\operatorname{upd}\left(((v, b), m), v^{\prime}\right)=\left(\operatorname{nxt}^{\prime}\left(e, m^{\prime}\right), \operatorname{upd}^{\prime}\left(m^{\prime}, \operatorname{nxt}^{\prime}\left(e, m^{\prime}\right)\right)\right)
$$

where $e=\left(v, v^{\prime}\right)$ and $m^{\prime}=\operatorname{upd}^{\prime}(m, e)$. Intuitively, the update-function mimics two moves in $\mathcal{A}^{\prime}$ : first, the one from $(v, b)$ to $e=\left(v, v^{\prime}\right)$ and then the move from this choice vertex determined by the strategy $\sigma^{\prime}$, which is given by $\operatorname{nxt}^{\prime}\left(e, m^{\prime}\right)$, where $m^{\prime}$ is the updated memory state.

Let $w$ be a play prefix of a play in $\mathcal{A}$. The memory state $\operatorname{upd}^{*}(w)=((v, b), m)$ encodes the following information: the simulated play $w^{\prime}$ in $\mathcal{A}^{\prime}$ ends in $(v, b)$, where $v$ is the last vertex of $w$, and we have $\operatorname{upd}^{\prime *}\left(w^{\prime}\right)=m$. Hence, it contains all information necessary to apply the next-move function nxt' to mimic $\sigma^{\prime}$. Hence, we define the next-move function nxt: $V_{0} \times M \rightarrow V$ for Player 0 in $\mathcal{A}$ by

$$
\operatorname{nxt}\left(v,\left(\left(v^{\prime}, b\right), m\right)\right)= \begin{cases}v^{\prime \prime} & \text { if } v=v^{\prime} \text { and } \operatorname{nxt}^{\prime}\left(\left(v^{\prime}, b\right), m\right)=\left(v^{\prime}, v^{\prime \prime}\right), \\ \bar{v} & \text { otherwise, for some } \bar{v} \in V \text { with }(v, \bar{v}) \in E .\end{cases}
$$

By definition of $\mathcal{M}$, the second case of the definition is never invoked, since $\operatorname{upd}^{*}(w v)=\left(\left(v^{\prime}, b\right), m\right)$ always satisfies $v=v^{\prime}$.

It remains to show that the strategy $\sigma$ implemented by $\mathcal{M}$ and nxt is indeed a winning strategy for Player 0 for $(\mathcal{A}, \varphi)$ with respect to some $\alpha$. To this end, let $k=(|V| \cdot|M|+3) \cdot W$ and define $\alpha(x)=2 k$ for every $x$, where $W$ is the largest weight in $\mathcal{A}$.

Let $\rho_{0} \rho_{1} \rho_{2} \cdots$ be a play in $\mathcal{A}$ that is consistent with $\sigma$. A straightforward induction shows that there exist bits $b_{0}, b_{1}, b_{2}, \cdots$ such that the play $\left(\rho_{0}, b_{0}\right)\left(\rho_{0}, \rho_{1}\right)\left(\rho_{1}, b_{1}\right)\left(\rho_{1}, \rho_{2}\right)\left(\rho_{2}, b_{2}\right) \cdots$ in $\mathcal{A}^{\prime}$ is consistent with $\sigma^{\prime}$. Hence, $w^{\prime \prime}=$ $\ell^{\prime}\left(\rho_{0}, b_{0}\right) \ell^{\prime}\left(\rho_{1}, b_{1}\right) \ell^{\prime}\left(\rho_{2}, b_{2}\right) \cdots$ satisfies $\varphi^{\prime}$. We show that $w^{\prime \prime}$ is $k$-bounded. This suffices to finish the proof as we can apply Lemma 32 and obtain $(\operatorname{tr}(\rho), \alpha) \models \varphi$, as $w^{\prime \prime}$ is a $k$-bounded coloring of $\operatorname{tr}(\rho)$. Thus, $\sigma$ is a winning strategy for Player 0 for $(\mathcal{A}, \varphi)$ with respect to $\alpha$.

Towards a contradiction assume that $w^{\prime \prime}$ is not $k$-bounded. Then, there exist positions $i<j$ such that

$-\rho_{i}=\rho_{j}$,

$-\operatorname{upd}^{\prime *}\left(\left(\rho_{0}, b_{0}\right) \cdots\left(\rho_{i}, b_{i}\right)\right)=\operatorname{upd}^{\prime *}\left(\left(\rho_{0}, b_{0}\right) \cdots\left(\rho_{j}, b_{j}\right)\right)$,

- the bits $b_{i}, \ldots, b_{j}$ are all equal, and

$-\operatorname{cst}\left(\rho_{i} \cdots \rho_{j}\right)>0$. 
To show this, one defines the sets $V_{j}$ of vertices visited between the $j$-th and the $(j+1)$-th edge with non-zero cost (including the $j$-th edge). This yields $|V| \cdot|M|+1$ non-empty sets of vertices of $(V \times\{0,1\}) \times M$ that coincide on the bit stored in their second component. Hence, we have derived the desired vertex repetition, as there are only $|V| \cdot|M|$ such vertices.

Hence, the play

$$
\rho^{*}=\left(\rho_{0}, b_{0}\right) \cdots\left(\rho_{i-1}, b_{i-1}\right)\left[\left(\rho_{i}, b_{i}\right) \cdots\left(\rho_{j-1}, b_{j-1}\right)\left(\rho_{j-1}, \rho_{j}\right)\right]^{\omega},
$$

obtained by traversing the cycle between $\left(\rho_{i}, b_{i}\right)$ and $\left(\rho_{j}, b_{j}\right)$ infinitely often, is consistent with $\sigma^{\prime}$, since the memory states reached at the beginning and the end of the loop are the same. Remember that the bits do not change between $i$ and $j$. Thus, $\operatorname{tr}\left(\rho^{*}\right)$ has only finitely many change points, but infinitely many occurrences of $\kappa$ and does therefore not satisfy $\chi$ under blinking semantics. This contradicts the fact that $\sigma^{\prime}$ is a winning strategy for $\left(\mathcal{A}^{\prime}, \operatorname{rel}(\varphi) \wedge \chi\right)$ under blinking semantics.

Now, we are able to prove Theorem 2 .

Proof. Hardness follows immediately from the 2ExPTIME-hardness of determining the winner of an LTL game [2021, as LTL is a fragment of cPLTL.

Membership in 2ExPTIME follows from the reductions described above: first, we turn the winning condition into a CPLTL $_{\mathbf{F}}$ formula and construct the LTL game under blinking semantics obtained from expanding the arena and relativizing the winning condition. This game is only polynomially larger than the original one and its winner (and a winning strategy) is computable in doublyexponential time.

By applying both directions of the proof of Lemma 7, we obtain a doublyexponential upper bound on the values of a satisfying variable valuation, if one exists. This is asymptotically tight, as one can already show doubly-exponential lower bounds for PROMPT-LTL [28].

Corollary 2. Fix a $c P L T L$ game $\mathcal{G}=(\mathcal{A}, \varphi)$ such that Player 0 has a winning strategy for $\mathcal{G}$ with respect to some $\alpha$. Then, Player 0 has a winning strategy for $\mathcal{G}$ with respect to a valuation that is bounded doubly-exponentially in the size of $\varphi$ and linearly in the number of vertices of $\mathcal{A}$ and the maximal cost in $\mathcal{A}$.

\section{Parametric LDL with Costs}

Linear Dynamic logic (LDL) 1425 extends LTL by temporal operators guarded with regular expressions, e.g., $\langle r\rangle \varphi$ holds at position $n$, if there is a $j$ such that $\varphi$ holds at position $n+j$ and the infix between positions $n$ and $n+j$ matches $r$. The resulting logic has the full expressiveness of the $\omega$-regular languages while retaining many of LTL's desirable properties like a simple syntax, intuitive semantics, a polynomial space algorithm for model checking, and a doubly-exponential time 
algorithm for solving games. Parametric LDL (PLDL) [15] allows to parameterize such operators, i.e., $\langle r\rangle_{\leq x} \varphi$ holds at position $n$ with respect to a variable valuation $\alpha$, if there is a $j \leq \alpha(x)$ such that $\varphi$ holds at position $n+j$ and the infix between positions $n$ and $n+j$ matches $r$. Model checking and solving games with PLDL specifications is not harder than for LTL, although PLDL is more expressive and has parameterized operators. In this section, we consider cPLDL where the parameters bound the cost of the infix instead of the length.

Formally, formulas of cPLDL are given by the grammar

$$
\begin{aligned}
& \varphi::=p|\neg p| \varphi \wedge \varphi|\varphi \vee \varphi|\langle r\rangle \varphi|[r] \varphi|\langle r\rangle_{\leq z} \varphi \mid[r]_{\leq z} \varphi \\
& r::=\phi|\varphi ?| r+r|r ; r| r^{*}
\end{aligned}
$$

where $p \in P, z \in \mathcal{V}$, and where $\phi$ ranges over propositional formulas over $P$. As for cPLTL, cPLDL formulas are evaluated on cost-traces with respect to variable valuations. Satisfaction of atomic formulas and of conjunctions and disjunctions is defined as usual, and for the four temporal operators, we define

- $(w, n, \alpha) \models\langle r\rangle \varphi$ if there exists $j \geq 0$ such that $(n, n+j) \in \mathcal{R}(r, w, \alpha)$ and $(w, n+j, \alpha) \models \varphi$,

- $(w, n, \alpha) \models[r] \varphi$ if for all $j \geq 0$ with $(n, n+j) \in \mathcal{R}(r, w, \alpha)$ we have $(w, n+$ $j, \alpha) \models \varphi$.

- $(w, n, \alpha) \models\langle r\rangle_{<z} \varphi$ if there exists $j \geq 0$ with $\operatorname{cst}\left(w_{n} c_{n} \cdots c_{n+j-1} w_{n+j}\right) \leq$ $\alpha(z)$ such that $(n, n+j) \in \mathcal{R}(r, w, \alpha)$ and $(w, n+j, \alpha) \models \varphi$,

$-(w, n, \alpha) \models[r]_{\leq z} \varphi$ if for all $j \geq 0$ with $\operatorname{cst}\left(w_{n} c_{n} \cdots c_{n+j-1} w_{n+j}\right) \leq \alpha(z)$ and with $(n, n+j) \in \mathcal{R}(r, w, \alpha)$ we have $(w, n+j, \alpha) \models \varphi$.

Here, the relation $\mathcal{R}(r, w, \alpha) \subseteq \mathbb{N} \times \mathbb{N}$ contains all pairs $(m, n) \in \mathbb{N} \times \mathbb{N}$ such that $w_{m} \cdots w_{n-1}$ matches $r$ and is defined inductively by

- $\mathcal{R}(\phi, w, \alpha)=\left\{(n, n+1) \mid w_{n} \models \phi\right\}$ for propositional $\varphi$,

$-\mathcal{R}(\psi ?, w, \alpha)=\{(n, n) \mid(w, n, \alpha) \models \psi\}$,

- $\mathcal{R}\left(r_{0}+r_{1}, w, \alpha\right)=\mathcal{R}\left(r_{0}, w, \alpha\right) \cup \mathcal{R}\left(r_{1}, w, \alpha\right)$,

$-\mathcal{R}\left(r_{0} ; r_{1}, w, \alpha\right)=\left\{\left(n_{0}, n_{2}\right) \mid \exists n_{1}\right.$ s.t. $\left(n_{0}, n_{1}\right) \in \mathcal{R}\left(r_{0}, w, \alpha\right)$ and $\left(n_{1}, n_{2}\right) \in$ $\left.\mathcal{R}\left(r_{1}, w, \alpha\right)\right\}$, and

$-\mathcal{R}\left(r^{*}, w, \alpha\right)=\{(n, n) \mid n \in \mathbb{N}\} \cup\left\{\left(n_{0}, n_{k+1}\right) \mid \exists n_{1}, \ldots, n_{k}\right.$ s.t. $\left(n_{j}, n_{j+1}\right) \in$ $\mathcal{R}(r, w, \alpha)$ for all $j \leq k\}$.

Again, we restrict ourselves to formulas where the set of variables parameterizing diamond operators and the set of variables parameterizing box operators are disjoint. Analogues of Lemma 1 and Lemma 2 hold for cPLDL, too.

The alternating-color is applicable to PLDL [15]: to this end, one introduces changepoint-bounded variants of the diamond- and the box-operator whose semantics only quantify over infixes with at most one changepoint. LDL formulas with changepoint-bounded operators can be translated into Büchi automata of exponential size. This allows to extend the algorithms for model-checking and realizability based on the alternating-color technique 18 to PLDL. Even more so, the algorithms presented in Section 4 and Section [5 can easily be adapted to cPLDL as well, again relying on the translation to Büchi automata via changepoint-bounded operators. 
Theorem 3. The cPLDL model checking problem is PSPACE-complete and determining the winner of games with cPLDL winning conditions is 2EXPTIMEcomplete.

\section{Multiple Cost Functions}

In this section, we consider parameterized temporal logics with multiple costfunctions. For the sake of simplicity, we restrict our attention to cPLTL, although all results hold for cPLDL, too.

Fix some dimension $d \in \mathbb{N}$. The syntax of mult-cPLTL is obtained by equipping the parameterized temporal operators by a coordinate $i \in\{1, \ldots, d\}$, denoted by $\mathbf{F}_{\leq_{i} x}$ and $\mathbf{G}_{\leq_{i} y}$. Here, a cost-trace is of the form $w_{0} \bar{c}_{0} w_{1} \bar{c}_{1} w_{2} \bar{c}_{2} \ldots$ where $w_{n} \in 2^{P}$ and $\bar{c}_{n} \in \mathbb{N}^{d}$. Thus, for every $i \in\{1, \ldots, d\}$, we can define

$$
\operatorname{cst}_{i}\left(w_{0} \bar{c}_{0} \cdots \bar{c}_{n-1} w_{n}\right)=\sum_{j=0}^{n-1}\left(\bar{c}_{j}\right)_{i}
$$

for every finite cost-trace $w_{0} \bar{c}_{0} \cdots \bar{c}_{n-1} w_{n}$. Furthermore, we require for every coordinate $i$ a proposition $\kappa_{i}$ such that $\kappa_{i} \in w_{n+1}$ if and only if $\left(\bar{c}_{n}\right)_{i}>0$.

The semantics of atomic formulas, boolean connectives, and unparameterized temporal operators are unchanged and for the parameterized operators, we define

- $(w, n, \alpha) \models \mathbf{F}_{\leq_{i} z} \varphi$ if and only if there exists a $j \geq 0$ with $\operatorname{cst}_{i}\left(w_{n} \bar{c}_{n} \cdots \bar{c}_{n+j-1} w_{n+j}\right) \leq \alpha(z)$ such that $(w, n+j, \alpha) \models \varphi$, and

- $(w, n, \alpha) \models \mathbf{G}_{\leq_{i} z} \varphi$ if and only if for every $j \geq 0$ with

$\operatorname{cst}_{i}\left(w_{n} \bar{c}_{n} \cdots \bar{c}_{n+j-1} w_{n+j}\right) \leq \alpha(z):(w, n+j, \alpha) \models \varphi$.

Again, we restrict ourselves to formulas where no variable parameterizes an eventually- and an always-operator, but we allow a variable to parameterize operators with different coordinates. Analogues of Lemma 1 and Lemma 2 hold for mult-cPLTL as well.

Example 2. A Streett condition with costs $\left(Q_{i}, P_{i}\right)_{i \in\{1, \ldots, d\}}$ [17] can be expressed in mult-cPLTL via

$$
\mathbf{F G}\left(\bigwedge_{i \in\{1, \ldots, d\}}\left(Q_{i} \rightarrow \mathbf{F}_{\leq_{i} x} P_{i}\right)\right) .
$$

In this setting, we consider the model checking problem for transition systems with $d$ cost functions and want to solve games in arenas with $d$ cost functions.

The alternating-color technique is straightforwardly extendable to the new logic mult-cPLTL: one introduces a fresh proposition $p_{i}$ for each coordinate $i$ and defines $\chi=\bigwedge_{i=1}^{d}\left(\left(\mathbf{G F} p_{i} \wedge \mathbf{G F} \neg p_{i}\right) \leftrightarrow \mathbf{G F} \kappa_{i}\right)$. Furthermore, the notions of $i$-blocks, $k$-boundedness in coordinate $i$, and $k$-spacedness in coordinate $i$ are defined as expected. Then, the proofs presented in Section 4 and Section 5 remain valid in this setting, too. 
In the case of model-checking, the third component of the colored Büchi graph $\mathcal{S} \times \mathfrak{A}$ has the form $2^{\left\{p_{1}, \ldots, p_{d}\right\}}$, i.e., it is exponential. However, this is no problem, as the automaton $\mathfrak{A}$ is already of exponential size. Similarly, in the case of infinite games, each vertex of the original arena has $2^{d}$ copies in $\mathcal{A}^{\prime}$, one for each element in $2^{\left\{p_{1}, \ldots, p_{d}\right\}}$ allowing Player 0 to produce appropriate colorings with the propositions $p_{i}$. The resulting game has an arena of exponential size (in the size of the original arena and of the original winning condition) and an LTL winning condition under blinking semantics. Such a game can still be solved in doubly-exponential time. To this end, one turns the winning condition into a deterministic parity automaton of doubly-exponential size with exponentially many colors, constructs the product of the arena and the parity automaton, which yields a parity game of doubly-exponential size with exponentially many colors. Such a game can be solved in doubly-exponential time [22].

Theorem 4. The mult-cPLTL model checking problem is PSPACE-complete and determining the winner of aninfinite game with mult-cPLTL winning condition is 2ExPTIME-complete.

Again, the same results hold for mult-cPLDL, which is defined as expected.

\section{Optimization Problems}

It is natural to treat model checking and solving games with specifications in parameterized linear temporal logics as an optimization problem: determine the optimal variable valuation such that the system satisfies the specification with respect to it. For parameterized eventualities, we are interested in minimizing the waiting times while for parameterized always', we are interested in maximizing the waiting times. Due to the undecidability results for not well-defined formulas one considers the optimization problems for the unipolar fragments, i.e., for formulas having either no parameterized eventualities or no parameterized always'.

In this section, we present algorithms for such optimization problems given by cPLTL specifications. For model checking, we are interested in the following four problems: given a transition system $\mathcal{S}$ and a $\mathrm{cPLTL}_{\mathbf{F}}$ formula $\varphi_{\mathbf{F}}$ and a $\mathrm{cPLTL}_{\mathbf{G}}$ formula $\varphi_{\mathbf{G}}$, respectively, determine

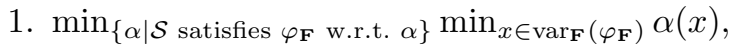

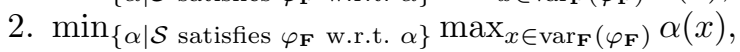

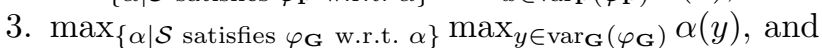

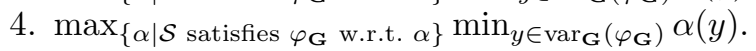

Applying the monotonicity of the parameterized operators and (in the first case) the alternating-color technique to all but one variable reduces the four optimization problems to ones where the specification has a single variable. Furthermore, the upper bounds presented in Corollary 1 and in Lemma 6 yield an exponential search space for an optimal valuation: if this space is empty, then 
there is no $\alpha$ such that $\mathcal{S}$ satisfies $\varphi_{\mathbf{F}}$ with respect to $\alpha$ in the first two cases respectively $\mathcal{S}$ satisfies $\varphi_{\mathbf{G}}$ with respect to every $\alpha$ in the latter two cases.

Thus, it remains the check whether the specification is satisfied with respect to some valuation that is bounded exponentially. In this setting, one can construct an exponentially sized non-deterministic Büchi automaton recognizing the models of the specification with respect to the given valuation (using a slight adaption of the construction presented in [28] accounting for the fact that we keep track of cost instead of time). This automaton can be checked for non-emptiness in polynomial space using an on-the-fly construction. Thus, an optimal $\alpha$ can be found in polynomial space.

Theorem 5. The cPLTL model checking optimization problems can be solved in polynomial space.

A similar approach works for infinite games as well. Here, we are interested in computing

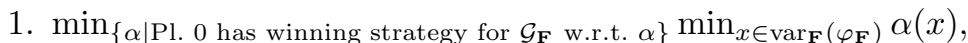

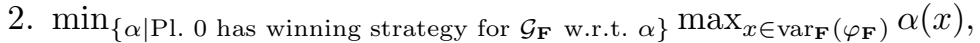

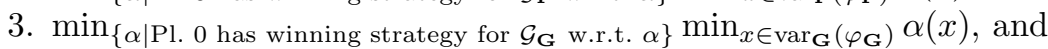

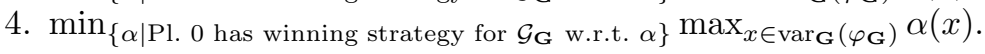

and witnessing winning strategies for given cPLTL games $\mathcal{G}_{\mathbf{F}}$ with $\mathrm{cPLTL}_{\mathbf{F}}$ winning condition $\varphi_{\mathbf{F}}$ and $\mathcal{G}_{\mathbf{G}}$ with cPLTL $_{\mathbf{G}}$ winning condition $\varphi_{\mathbf{G}}$.

Again, one can reduce these problems to the case of winning conditions with a single variable and by applying determinacy of games with respect to a fixed valuation, it even suffices to consider the case of CPLTL $_{\mathbf{F}}$ winning conditions with a single variable, due to duality of games: swapping the players in a game with cPLTL $_{\mathbf{G}}$ winning condition yields a game with CPLTL $_{\mathbf{F}}$ winning condition. Corollary 2 gives a doubly-exponential upper bound on an optimal variable valuation. Hence, one can construct a deterministic parity automaton of triply-exponential size with exponentially many colors recognizing the models of the specification with respect to a fixed variable valuation $\alpha$ that is below the upper bound (again, see 28] for the construction). Player 0 wins the parity game played in the original arena but using the language of the automaton as winning condition if and only if she has a winning strategy for the CPLTL $_{\mathbf{F}}$ game with respect to $\alpha$. Such a parity game can be solved in triply-exponential time [22]

Theorem 6. The cPLTL optimization problems for infinite games can be solved in triply-exponential time.

Furthermore, the same results hold for cPLDL using appropriate adaptions of the automata constructions presented in [15]16].

Theorem 7. The cPLDL model checking optimization problems can be solved in polynomial space and the cPLDL optimization problems for infinite games can be solved in triply-exponential time. 
However, for parameterized logics with multiple cost-functions, these results do not remain valid, as one cannot reduce the optimization problems to ones with a single variable, as a variable may bound operators in different dimensions. Thus, one has to keep track multiple costs, which incurs an exponential blow-up when done naively. Whether this can be improved is an open question.

\section{Conclusion}

We introduced parameterized temporal logics whose operators bound the accumulated cost instead of time as usual: cPLTL and cPLDL as well as their variants mult-cPLTL and mult-cPLDL with multiple cost functions retain the attractive algorithmic properties of LTL like a polynomial space model checking algorithm and a doubly-exponential time algorithm for solving infinite games. Even the optimization variants of these problems are not harder for cPLTL and cPLDL than for PLTL: polynomial space for model checking and triply-exponential time for solving games. However, it is open whether these problems are harder for logics with multiple cost functions. Another open question concerns the complexity of the optimization problem for infinite games: can these problems be solved in doubly-exponential time, i.e., is finding optimal variable valuations as hard as solving games? Note that this question is already open for PLTL. Finally, one could consider weights from some arbitrary semiring and corresponding weighted parameterized temporal logics.

\section{References}

1. Alur, R., Etessami, K., Torre, S.L., Peled, D.: Parametric temporal logic for "model measuring". ACM Trans. Comput. Log. 2(3), 388-407 (2001)

2. Bloem, R., Chatterjee, K., Henzinger, T.A., Jobstmann, B.: Better quality in synthesis through quantitative objectives. In: Bouajjani, A., Maler, O. (eds.) CAV. LNCS, vol. 5643, pp. 140-156. Springer (2009)

3. Bojańczyk, M.: A bounding quantifier. In: Marcinkowski, J., Tarlecki, A. (eds.) CSL. LNCS, vol. 3210, pp. 41-55. Springer (2004)

4. Bojańczyk, M.: Weak MSO with the unbounding quantifier. Theory Comput. Syst. 48(3), 554-576 (2011)

5. Bojanczyk, M.: Weak MSO+U with path quantifiers over infinite trees. In: Esparza, J., Fraigniaud, P., Husfeldt, T., Koutsoupias, E. (eds.) ICALP Part II. LNCS, vol. 8573, pp. 38-49. Springer (2014)

6. Bojańczyk, M., Colcombet, T.: Bounds in $\omega$-regularity. In: LICS. pp. 285-296. IEEE Computer Society (2006)

7. Bojańczyk, M., Toruńczyk, S.: Weak MSO+U over infinite trees. In: Dürr, C., Wilke, T. (eds.) STACS. Leibniz International Proceedings in Informatics (LIPIcs), vol. 14, pp. 648-660. Schloss Dagstuhl-Leibniz-Zentrum für Informatik, Dagstuhl, Germany (2012)

8. Brázdil, T., Chatterjee, K., Kucera, A., Novotný, P.: Efficient controller synthesis for consumption games with multiple resource types. In: Madhusudan, P., Seshia, S.A. (eds.) CAV. LNCS, vol. 7358, pp. 23-38. Springer (2012) 
9. Č tative synthesis for concurrent programs. In: Gopalakrishnan, G., Qadeer, S. (eds.) CAV. LNCS, vol. 6806, pp. 243-259. Springer (2011)

10. Chatterjee, K., Doyen, L.: Energy parity games. In: Abramsky, S., Gavoille, C., Kirchner, C., Meyer auf der Heide, F., Spirakis, P.G. (eds.) ICALP (2). LNCS, vol. 6199, pp. 599-610. Springer (2010)

11. Chatterjee, K., Henzinger, T.A., Horn, F.: Finitary winning in omega-regular games. ACM Trans. Comput. Log. 11(1) (2009)

12. Chatterjee, K., Henzinger, T.A., Jurdziński, M.: Mean-payoff parity games. In: LICS. pp. 178-187. IEEE Computer Society (2005)

13. Colcombet, T.: The theory of stabilisation monoids and regular cost functions. In: Albers, S., Marchetti-Spaccamela, A., Matias, Y., Nikoletseas, S.E., Thomas, W. (eds.) ICALP Part II. LNCS, vol. 5556, pp. 139-150. Springer (2009)

14. De Giacomo, G., Vardi, M.Y.: Linear temporal logic and linear dynamic logic on finite traces. In: Rossi, F. (ed.) IJCAI. IJCAI/AAAI (2013)

15. Faymonville, P., Zimmermann, M.: Parametric linear dynamic logic. In: Peron, A., Piazza, C. (eds.) GandALF. EPTCS, vol. 161, pp. 60-73 (2014)

16. Faymonville, P., Zimmermann, M.: Parametric linear dynamic logic (full version). CoRR abs/1504.03880 (2015), http://arxiv.org/abs/1504.03880

17. Fijalkow, N., Zimmermann, M.: Parity and streett games with costs. Logical Methods in Computer Science 10(2) (2014)

18. Kupferman, O., Piterman, N., Vardi, M.Y.: From liveness to promptness. Formal Methods in System Design 34(2), 83-103 (2009)

19. Leucker, M., Sánchez, C.: Regular linear temporal logic. In: Jones, C., Liu, Z., Woodcock, J. (eds.) ICTAC'07. LNCS, vol. 4711, pp. 291-305. Springer-Verlag, Macau, China (September 2007)

20. Pnueli, A., Rosner, R.: On the synthesis of a reactive module. In: POPL. pp. 179190 (1989)

21. Pnueli, A., Rosner, R.: On the synthesis of an asynchronous reactive module. In: Ausiello, G., Dezani-Ciancaglini, M., Rocca, S.R.D. (eds.) ICALP. LNCS, vol. 372, pp. 652-671. Springer (1989)

22. Schewe, S.: Solving parity games in big steps. In: Arvind, V., Prasad, S. (eds.) FSTTCS. LNCS, vol. 4855, pp. 449-460. Springer (2007)

23. Sistla, A.P., Clarke, E.M.: The complexity of propositional linear temporal logics. J. ACM 32(3), 733-749 (1985)

24. Vanden Boom, M.: Weak cost monadic logic over infinite trees. In: Murlak, F., Sankowski, P. (eds.) MFCS. LNCS, vol. 6907, pp. 580-591. Springer (2011)

25. Vardi, M.Y.: The rise and fall of LTL. In: D'Agostino, G., Torre, S.L. (eds.) GandALF. EPTCS, vol. 54 (2011)

26. Vardi, M.Y., Wolper, P.: Reasoning about infinite computations. Inf. Comput. 115(1), 1-37 (1994)

27. Wolper, P.: Temporal logic can be more expressive. Information and Control $56(1-2), 72-99(1983)$

28. Zimmermann, M.: Optimal bounds in parametric LTL games. Theor. Comput. Sci. 493, 30-45 (2013) 